\title{
ROAD NETWORK MAPPING USING AIRBORNE LiDAR DATA
}

\author{
Suryakant Upadhayay ${ }^{1}$, Manohar Yadav ${ }^{1, *}$, Dheerendra Pratap Singh ${ }^{1}$ \\ ${ }^{1}$ Motilal Nehru National Institute of Technology Allahabad, India - suryathekingg@gmail.com, ssmyadav@mnnit.ac.in, \\ dheeru.dp@gmail.com
}

\section{Commission V, SS: Emerging Trends in Remote Sensing}

KEYWORDS: Road, Airborne Laser Scanning (ALS), Intensity, Maximum Likelihood (ML) Classification, Digital Terrain Model (DTM), Digital Surface Model (DSM)

\begin{abstract}
:
The accurate, detailed and up-to-date road information is highly essential geo-spatial databases for transportation, smart city and other related applications. Thus, the main objective of this research is to develop an efficient algorithm for road network extraction from airborne LiDAR data using supervised classification approach. The proposed algorithm first classifies the input data into the road and non-road features using modified maximum likelihood classification approach. Then Digital Terrain Model (DTM) mask is generated by removing non-ground features from Digital Surface Model using hierarchical morphology and road candidate image if obtained. The parking lots are removed and road network is extracted successfully.
\end{abstract}

\section{INTRODUCTION}

The road network often gets modified due to various developmental activities and therefore an upgraded map of road is helpful for the various applications. Some of those applications are transportation management, safety analysis, situational awareness, mission planning and decision making in emergency planning. The manual delineation of road features from imagery is labour intensive and tedious work which requires highly skilled technical personnel, large attention to details and a scrupulous commitment to quality and completeness.

The various approaches have been developed for the automatic road network extraction but still their accuracy, completeness and automation are a challenge among the researchers.

In the past, only aerial and satellite imageries were used for the road network extraction. The extracted network lacks in the accuracy and completeness due to non-availability of depth information in the input data that helps to separate road and its surrounding. The recent advancement in LiDAR technology makes it feasible to acquire the dense point cloud which gives very detailed information. The LiDAR point cloud although provides rich information about the scene but the data size is very large, which demands heavy computational resources for the processing. Therefore, an approach is presented in this paper that uses intensity and depth information for rapid extraction of road features from LiDAR point cloud.

The researchers were tried to delineate roads from input remote sensing data like LiDAR data. The method of Zhao et al. (2012) removed the non-ground points from image and then applied EM classification to generate the road candidate image. They performed direction based cumulative voting to remove parking lots and used geometrically guided gap filling. Youn et al. (2008) used orientation based image segmentation and region based acupuncture method to generate road candidate image. Péteri et al. (2003) used specific active contours (snakes) combined with multi-resolution analysis for the reconstruction of roads. Clode et al. (2004) used minimum and maximum intensity of roads as a threshold for filtering roads from LiDAR data of ground surface only. Samadzadegan et al. (2009) used classifier fusion approach to classify LiDAR points into road or non-road points using the multi-classifier system.

Zhang et al. (2006) designed an approach, which used angular texture signature of the imagery to remove parking lots from the road features. Shackelford et al. (2003) used high resolution multispectral satellite imagery and performed land cover classification using pixel based fuzzy classification approach. They further used object based classification approach for the refinement of result. They grew line segments in the urban land cover classification to trace the roads.

The road networks mapping from aerial or satellite images generally retain less information about road surfaces and incomplete data compared to the LiDAR data. LiDAR data is volumetric and their processing need extra computational resources compare to images. Method of Zhao et al. (2012) used intensity and depth images of airborne laser scanning (ALS) data to efficiently detect the road network.

The road network extraction methodology proposed in this research paper uses intensity and depth images generated from LiDAR data. The modified maximum likelihood classification technique is used to identify roads, which is faster and less complex. Thereafter, the parking lots are removal followed by extraction of road centrelines.

\footnotetext{
* Corresponding author
} 


\section{METHODOLOGY}

An algorithm consisting of three modules is designed for extraction and characterization of road network using ALS data (see Figure 1). In the first module, pixel based modified maximum likelihood (ML) image classification is performed. The second module consists of generation of digital terrain model (DTM) mask and its integration with classified image obtained from modified ML classification in the first module.

Third module consists of parking lots removal using morphological operations and generation of road network.

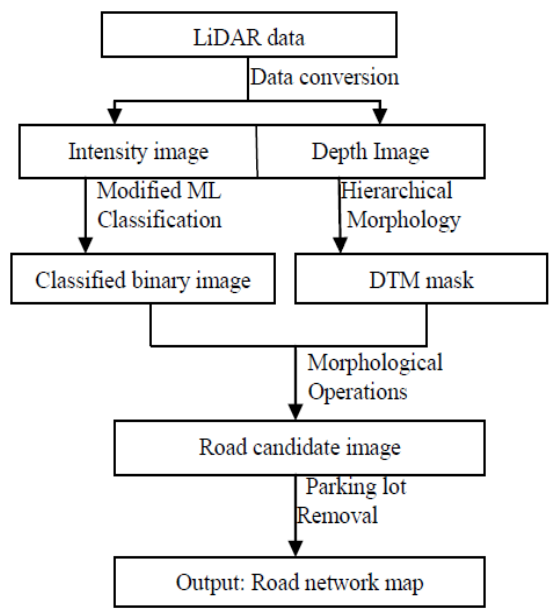

Figure 1. Workflow of the proposed algorithm

In the following sections the different modules of the algorithm are described in detail.

\subsection{Classification}

Input ALS data is used to generate 8-bit intensity image from the intensity values. The modified maximum likelihood classification technique is applied on intensity image to generate separate road from non-road pixels. This approach reduces the complexity as well as computational time during the processing. The proposed approach assumes that the distribution of pixels value forming a separate class in the intensity image is Gaussian. Therefore, Gaussian probability density function is used to represent each class as the likelihood function. The Gaussian likelihood function is given in given below:

$$
L_{k}(X)=\frac{1}{\left(\sigma_{k} \sqrt{2 \pi}\right)} \exp \left(-\frac{\left(X-\mu_{k}\right)^{2}}{2 \sigma_{k}^{2}}\right)
$$

Where $X$ is an image pixel, $L_{k}(X)$ is likelihood of $X$ belonging to class $\mathrm{k}, \mu_{k}$ is mean of class $\mathrm{k}, \sigma_{k}$ is standard deviation of class $k$.

$$
\operatorname{Mean}(\mu)=\frac{1}{n} \sum_{j=1}^{n} X_{j}
$$

$$
\operatorname{Variance}\left(\sigma^{2}\right)=\frac{1}{n} \sum_{i=1}^{n}\left(X_{i}-\mu\right)^{2}
$$

This classification approach divides the intensity image into two classes, that is, road and non-road. Using this classification technique, the classified image created is a binary image where foreground pixel represents road class and background pixels represents non road class. The modified ML classification algorithm is explained in detail in subsequent sections-

(1) Intensity image is taken as the input for modified ML classification

(2) Training samples from roads of different intensity values are selected to represent majority of road surfaces in urban areas. Since the image has to be classified in only two classes i.e. road and non-road, hence training samples from roads of different intensity values are chosen. Intensity values of LiDAR data of roads are not same due to different materials used in wearing course of the road and their different reflective properties. Sample data of different road class have been chosen for identifying roads based on intensity values. Five samples of roads are taken as training samples and named as road classes 1 to 5 , which represents majority of road surfaces in urban areas.

(3) The mean, standard deviation of each class are calculated.

(4) Threshold value of intensity variation i.e. value of $\left(X-\mu_{k}\right)$ is calculated. From the selected training samples, maximum intensity value $\max _{i}$ and minimum intensity value $\min _{i}$ are calculated. Minimum value among $\max _{i}$ and $\min _{i}$ are then calculated. This minimum value is stored as $X$. Thus, threshold is defined as $\left(X-\mu_{k}\right)$. The likelihood function $\mathrm{L}_{\mathrm{k}}(\mathrm{X})$ gains its maximum value for $\left(X-\mu_{k}\right)=0$ and for calculating minimum value, above defined threshold is used.

Minimum and maximum likelihood values $\left(\mathrm{L}_{\mathrm{k}}\right)$ of each class are calculated using equation (1).

This process is repeated for each pixel in the image

(5.1) The next pixel value is chosen

(5.2) The likelihood function value of that pixel is calculated by using mean and standard deviation of road class -1 using equation (1). If likelihood function value of the pixel lies between minimum and maximum likelihood value of road class-1 then set the Digital Number (DN) value of the pixel as 1 , indicating that the pixel belongs to road class and then go to step (5.1). This is done to increase speed of classification, because, if pixel belong to any one road class then we don't have to check for next road class as it is already classified as road pixel. If likelihood function value of the pixel does not lies between the minimum and maximum range of the road class- 1 then it is checked for road class-2 and so on and go to step (5.1) 
immediately, by setting pixel DN value as 1 , if pixel belongs to any road class.

If the pixel does not belong to any road class then set its $\mathrm{DN}$ value as 0 indicating that this is non-road pixel and then go to step (5.1)

(5.3) If all pixels are classified then classification process is completed

Once the above classification is performed a binary image is obtained.

Accuracy of classification depends upon training samples chosen. The concrete roof material of buildings has similar reflective properties as of a cement concrete roads hence these roof tops are also present in road class. This is the error of commission. Some other non-ground features e.g. trees are also present in the classification result as error of commission. Figure 2 and Figure 3 shows the intensity image used and our classification result respectively. In the subsequent modules these errors are removed to get the road candidate image.

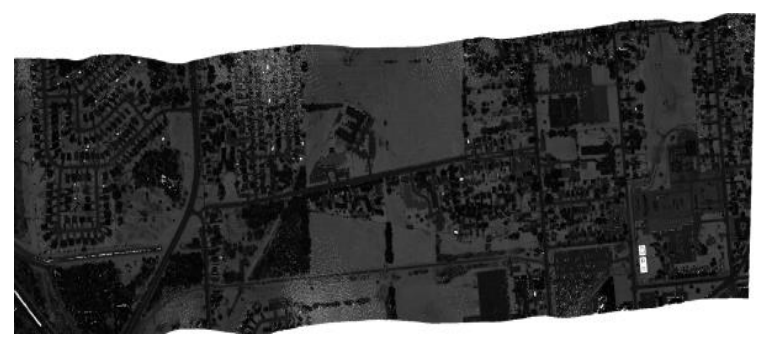

Figure 2. Intensity image

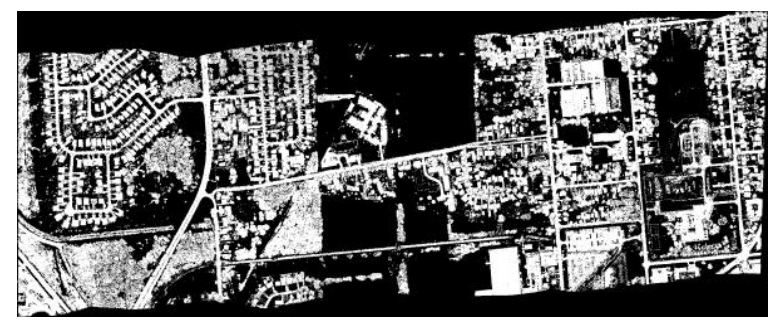

Figure 3. Classification result

\subsection{Generation of the road candidate image}

In this module, a binary mask (i.e. DTM mask) is generated which contains only ground features as foreground objects and non-ground features as background objects. This mask is used with the classification result obtained in previous module to remove all non-ground features which are present in the classification result also known as error of commission. This includes buildings etc. For generating DTM mask hierarchical morphological operations on depth image are performed. After removing non-ground features from classification result, morphological area opening for 8-connected components is applied to filter small erroneous objects thus road candidate image is obtained.

The DTM mask generation algorithm is explained in detail in subsequent sections-

(1) The structuring element (SE) of a particular shape, such as disk or octagon is taken. Set its maximum and minimum size as $\mathrm{SE}_{\max }, \mathrm{SE}_{\min }$. An increasing step $\mathrm{x}$ is defined, after each iteration its size is increased by $\mathrm{x}$, i.e. $\mathrm{SE}_{\mathrm{k}+1}=\mathrm{SE}_{\mathrm{k}}+\mathrm{x}$. initially set $\mathrm{SE}_{1}=\mathrm{SE}_{\text {min }}$.

(2) At the $\mathrm{k}^{\text {th }}$ iteration, to get approximate digital terrain model DTM , the morphological opening is performed on depth image with size of structuring element $\mathrm{SE}_{\mathrm{k}}$. Since depth image is a gray scale image hence the gray scale morphological opening is used. To identify elevated pixels, we set a threshold $\mathrm{T}_{\mathrm{k}}$. Elevated pixels are those which has elevation difference greater than threshold $\mathrm{T}_{\mathrm{k}}$, i.e. $\left|\mathrm{DSM}_{\mathrm{k}-1}(\mathrm{i})-\mathrm{DTM}_{\mathrm{k}}(\mathrm{i})\right|>\mathrm{T}_{\mathrm{k}}$.

(3) All elevated pixels are grouped in 8-connected components. Those components having area greater than AreasEk -1 classified as elevated objects i.e. nonterrain $_{\mathrm{k}}$. These non-terrain objects are removed from DSM so that they need not be processed again in subsequent iterations. Now set $\mathrm{DSM}_{\mathrm{k}}=\mathrm{DSM}_{\mathrm{k}-1}-$ nonterraink.

(4) Repeat step 2 and step 3 iteratively until $\mathrm{SE}_{\mathrm{k}}>\mathrm{SEmax}$. All elevated objects are obtained as output.

(5) Result generated in step 4 is a binary image where foreground pixel corresponds to non-terrain objects. Take complement of this image to generate DTM mask.

(6) The algorithm ends and output is obtained.

In above algorithm, $\mathrm{DSM}_{\mathrm{k}}$ is the digital surface model after $\mathrm{k}^{\text {th }}$ iteration and $\mathrm{DTM}_{\mathrm{k}}$ is digital terrain model after $\mathrm{k}^{\text {th }}$ iteration. $\mathrm{SE}_{\mathrm{k}}$ denotes the size of structuring element at $\mathrm{k}^{\text {th }}$ iteration. Areasek-1 is the area of structuring element with size $\mathrm{SE}_{\mathrm{k}}$. DTMk(i) and $\mathrm{DSM}_{\mathrm{k}}(\mathrm{i})$ is the value of pixel ' $\mathrm{i}$ ' on $\mathrm{DTM}_{\mathrm{k}}$ and $\mathrm{DSM}_{\mathrm{k}}$, respectively.

Figure 4, Figure 5 and Figure 6 represents depth image used, DTM mask generated and road candidate image generated at the end of this module. As we can see that road candidate image now does not have any error of commission except parking lots. In the next module, parking lots are also removed and refined road network map is obtained.

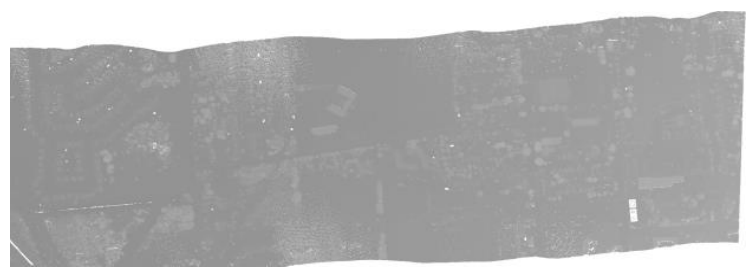

Figure 4. Depth image 


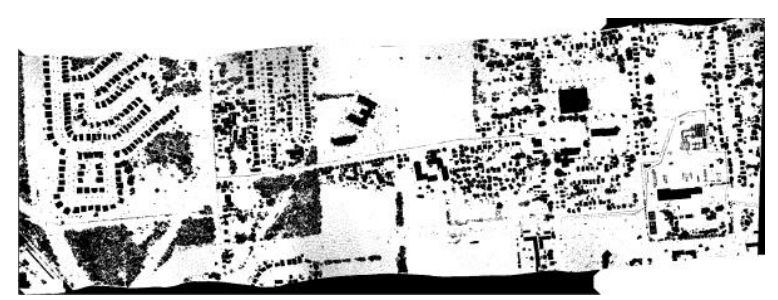

Figure 5. DTM mask

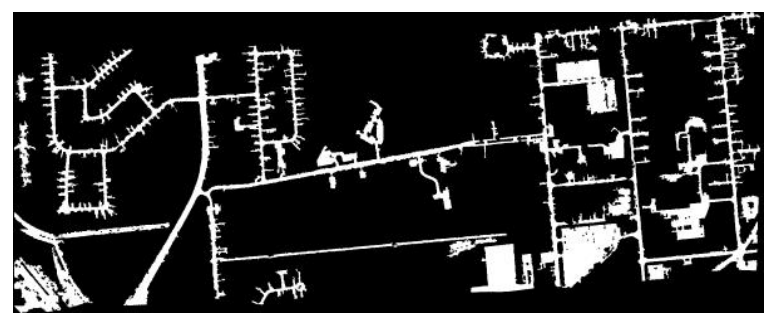

Figure 6. Road candidate image

\subsection{Elimination of parking lots}

For removing parking lots, the standard roadway widths, as specified by various codes, are considered. Parking lots have been eliminated by performing morphological operations on the road candidate image. Using binary road candidate image most of the parking lots are removed but it is not possible to remove them completely as they are connected to roads.

Due to parked vehicles in the parking lots or due to the presence of trees, there are some holes (pixel gaps) in the road candidate image as if it is a non-ground point. It creates problems in removing parking lots. Thus it is advisable to perform morphological closing on the road candidate image to fill those holes. Some of those holes are of bigger size and does not get filled properly.

The stepwise procedure for parking lots removal algorithm is given below-

(1) Firstly, all standard carriageway widths of roads, specified in various codes, are defined.

(2) The size of structuring element is determined on the basis of maximum acceptable width of the road as per standards. Size and shape of structuring element plays an important role. Preferably it should be square or rectangle for better results.

(3) Morphological opening is performed on the road candidate image with the selected structuring element.

(4) Complement of the result of step 3 is obtained.

(5) Perform Logical AND operation between road candidate image and result of step 4 .

(6) Perform morphological area opening with appropriate threshold for 8- connected components. This is done to clean the image and filter out small disconnected components.

Figure 7 shows the final road network map generated after this algorithm.
Now, the centerlines of the roads are extracted. Morphological operations are used to extract this. Skeleton of the image is determined and then short extraneous spurs are removed. They are also known as parasitic components, to get the result. Figure 8 shows the extracted centerlines of the roads.

\section{EXPERIMENT}

\subsection{Test Data}

The developed algorithm has been applied on sample airborne LiDAR dataset of Niagara Falls area, in USA. Size of the LiDAR data set is $118 \mathrm{MB}$. The LiDAR point cloud data is converted into depth and intensity images using $1 \mathrm{~m}$ resampling distance in both $\mathrm{x}$ and $\mathrm{y}$ direction. Thus intensity image and depth image of sizes approx $4.18 \mathrm{MB}$, having dimensions $1932 \times 758$ pixels are obtained.

\subsection{Result}

The extracted road network is shown in Figure 7 and Figure 8 shows the centerlines of the roads. These results are analyzed quantitatively as presented in subsequent sections.

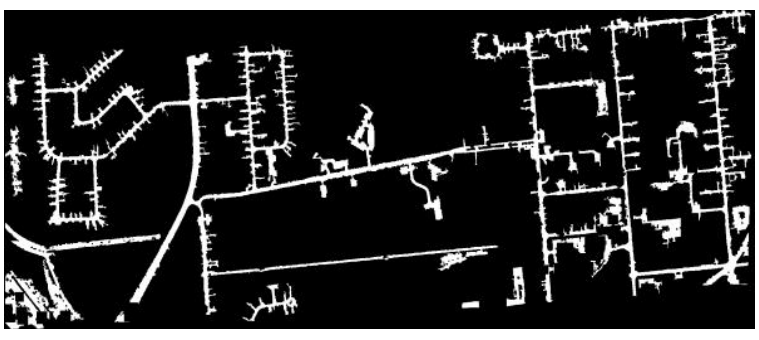

Figure 7. Resultant road network map

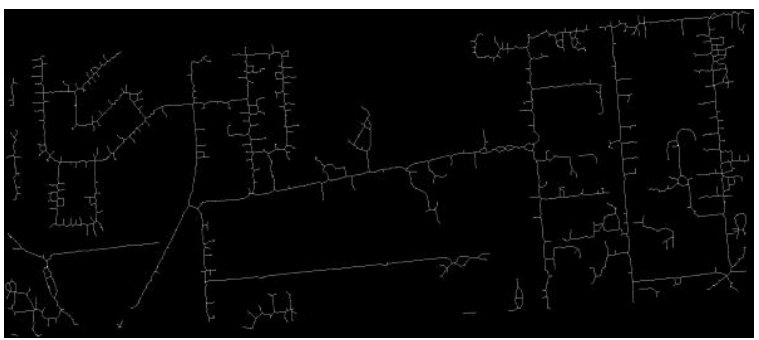

Figure 8. Road centerlines

\subsection{Quantitative evaluation of road extraction}

The actual road network of the test area is obtained by manually digitizing the intensity image. All kinds of roads (public and private) were considered in ground truth data except the car parking. As discussed in Zhao et al. (2012) accuracy and completeness of the methodology is evaluated by calculating the following parameters- 


$$
\begin{gathered}
\text { Accuracy }=\mathrm{R}_{\mathrm{C}} / \mathrm{R}_{\mathrm{A}} \\
\text { Completeness }=\mathrm{R}_{\mathrm{A}} / \mathrm{R}_{\mathrm{T}}
\end{gathered}
$$

In the above equations $\mathrm{R}_{\mathrm{T}}$ is the total length of the manually digitized roads, $R_{\mathrm{A}}$ is the total length of the automatically extracted roads using the above algorithms, and $R_{C}$ is the total length of the extracted roads that match the manually determined roads. Figure 9 shows the ground truth data after digitization.

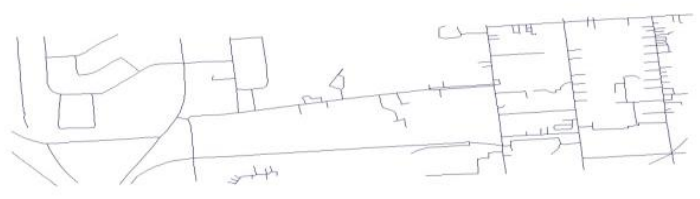

Figure 9. Ground truth data

In our experiment we have got accuracy of $94 \%$ and completeness of $88 \%$.

\subsection{Sensitivity of the result}

Classification results are dependent on the intensity variation threshold chosen and training samples chosen. Result of DTM mask generation algorithm is dependent on the sizes of structuring elements chosen and elevation difference threshold $\left(T_{k}\right)$. In the developed algorithm $T_{k}$ is taken as 3. Parking lot removal algorithm is dependent mainly on parameter structuring element's size. Completeness will depend on the training area selection for the classification and varies from scene to scene.

\section{CONCLUSIONS AND FUTURE WORK}

This paper describes effective, easy to implement and fast approach to road network extraction. The various existing algorithms have been studied and their limitations were analysed. The results of the proposed method is found accurate and more importantly it fairly simple to implement in any programming languages. The car parking areas are also removed in the developed methodology. The presence of shadows of building or trees on roads reduces the completeness measure of this method.

Future work includes designing of new approach that can deal with shadows of other objects on the road and objects lying on the road surfaces.

\section{ACKNOWLEDGEMENTS}

The authors acknowledge the Optech Inc., Canada, which is now Teledyne Optech Inc., for sharing the ALS data.

\section{REFERENCES}

Chabat, F., Yang, G.Z., Hansell, D.M., 1999. A corner orientation detector. Image and vision computing, 17(10), pp. 761-769.

Clode, S., Kootsookos, P., Rottensteiner, F., 2004. The Automatic extraction of Roads from LiDAR data. In: Proc. of ISPRS 20th Annual Congress, vol. 35, pp. 231-236.

Hu, X., Tao, C.V., Hu, Y., 2004. Automatic Road Extraction from dense urban area by integrated processing of high resolution imagery and LiDAR data. In: The International Archives of the Photogrammetry, Remote Sensing and Spatial Information Sciences, Vol. XXXV Part B3, pp. 320-324.

Péteri, R., Celle, J., Ranchin, T., 2003. Detection and Extraction of Road Networks from high resolution satellite images. In: Proc. of the IEEE International Conference on Image Processing (ICIP'03), Barcelona, pp. 301-304.

Samadzadegan, F., Hahn, M., Bigdeli, B., 2009. Automatic road extraction from LIDAR data based on classifier fusion. In: Proc. of Urban Remote Sensing Event, Shanghai, pp. $1-6$.

Shackelford, A.K., Davis, C.H., 2003. Urban road network extraction from high-resolution multispectral data. In: $2 n d$ GRSS/ISPRS Joint Workshop on Remote Sensing and Data Fusion over Urban Areas, Berlin, Germany, pp. 142 - 146.

Youn, J., Bethel, J. S., Mikhail, E. M., Lee, C., 2008. Extracting Urban Road Networks from high-resolution true Orthoimage and LiDAR. Photogrammetric Engineering \& Remote Sensing, 74(2), pp. 227-237.

Zhang, Q., Couloigner, I., 2006. Benefit of the angular texture signature for the separation of parking lots and roads on high resolution multi-spectral imagery. Pattern Recognition Letters, 27(9), pp. 937-946.

Zhao, J., You, S., Huang, J., 2012. Rapid Extraction and Updating of Road Network from Airborne LiDAR Data. In: Proceedings of the 2011 IEEE Applied Imagery Pattern Recognition Workshop (AIPR), pp. 1-7. 